\title{
Ontología social y práctica jurídica: un debate
}

\author{
Giovanni Tuzet ${ }^{*}$ \\ (Traducción del inglés de Diego Dei Vecchi)
}

Recepción y evualación de propuesta: 1/7/2013

Aceptación: 1/10/2013

Recepción y aceptación final 3/6/14

\begin{abstract}
Resumen: La presente discusión reúne cuatro textos que discuten sobre una cuestión central para los teóricos del derecho. En efecto, si se considera que el derecho es una práctica social, en qué sentido ello se vincula con diferentes teorías del derecho (Austin, Hart, Shapiro) y cuál de ellas puede dar cuenta mejor de lo que usualmente se asocia al derecho.
\end{abstract}

Palabras clave: derecho, práctica social, acción grupal.

\begin{abstract}
The present debate gathers four papers discussing a central question in legal theory. In effect, the discussion regarding if and in what sense the law is a social practice, the proper relation between different theories of law (Austin, Hart, Shapiro), and which among them gives the proper account of what it is normally associated to the concept of law.
\end{abstract}

Key words: law, social practice, joint action.

El presente número de esta revista acoge de manera encomiable un debate sobre la ontología social del derecho. Se trata de un debate generado por el artículo de Carlos Bernal Austin, Hart y Shapiro: tres variaciones alrededor del derecho como una entidad fundada en una práctica social. Dos académicos, Damiano Canale y Richard Ekins, comentaron tal artículo, en tanto que Bernal aceptó escribir algunas réplicas a sus

* Universidad Bocconi (Milán, Italia). 
comentarios críticos. En esta breve introducción trato de arrojar alguna luz sobre los que considero los aspectos principales del debate.

Como editor de la discusión, me gustaría poner de manifiesto que el debate concierne básicamente a lo que llamaré la ontología-proceso. Como una entidad, o mejor, un conjunto de entidades, el derecho es ontológicamente distinto respecto del proceso que lo genera. De modo que si queremos indagar acerca de la naturaleza del derecho, debemos distinguir la ontología-proceso de la ontología-resultado (u ontología-entidad si se prefiere). En mi opinión, las intenciones y los actos colectivos pertenecen al reino de lo primero. Las reglas y los procedimientos jurídicos pertenecen a lo segundo. Ello es así dado que una cosa son las condiciones bajo las cuales el derecho es producido, y otra muy distinta el derecho en sí, aun cuando ambas cuestiones están estrechamente relacionadas por el simple hecho de que la primera produce a la segunda ${ }^{1}$. Kelsen lo dice de este modo:

"El acto creador de la norma es un hecho que existe en el tiempo y el espacio y que puede ser percibido por nuestros sentidos. Este hecho puede ser descripto mediante un enunciado del ser. Pero este hecho es diferente respecto a su significado - esto es, la norma - que es el objeto de la teoría del derecho, y que no puede ser descripto mediante un enunciado del ser, sino solamente a través de un enunciado de deber ser ${ }^{2}$.

En sentido similar, el proceso de escritura de un poema es distinto a - aunque está estrechamente relacionado con- el poema mismo.

Por una parte, debe ponerse el foco de atención en el proceso de creación y mantenimiento de una sociedad política o una comunidad dotada de un sistema jurídico. Por la otra, debe apuntarse a los resultados de ese proceso y en particular a las reglas y las disposiciones que establece para la vida de la sociedad o la comunidad. Qué se

1 He subrayado ya este punto al discutir el convencionalismo de Marmor, en Tuzet, G., "The Social Reality of Law", en Analisi e diritto, 2007, págs. 179198; en particular, págs. 189, 194.

2 Kelsen, H., "What is the Pure Theory of Law", Tulane Law Review, 34, 1960, pág. 271. 
encuentra primero - desde el punto de vista conceptual- es difícil de decir. Se trata de una forma del problema o la paradoja del "huevo y la gallina"3. Las gallinas no pueden existir sin huevos de gallina, y viceversa. Las instituciones jurídicas no pueden existir sin normas jurídicas confiriendo a ellas algunos poderes jurídicos, y viceversa: las normas jurídicas no pueden existir sin instituciones jurídicas o autoridades que las creen. Pero desde un punto de vista explicativo asumo que las cosas son menos problemáticas en nuestro caso que en el de las gallinas: el proceso social generador de derecho es explicativamente prioritario respecto de los resultados que produce.

Tradicionalmente la teoría del derecho ha estado preocupada por la ontología-resultado. Una pregunta como "¿qué es una norma jurídica válida?” es un típico ejemplo de ello. Ahora bien, para volver sobre el punto, pienso que el presente debate atañe principalmente a la ontología-proceso del derecho, esto es, a las condiciones bajo las cuales el derecho es generado. Por lo tanto, no ha de buscarse aquí una aproximación ontológica al derecho si uno está interesado en la ontología-resultado. Esta discusión constituye más bien un estudio fundamental acerca de las condiciones sociales de aquella.

El artículo de Bernal comienza resaltando que la realidad social es diferente de la realidad natural (una ley es ontológicamente distinta a un tigre, en el sentido de que pertenecen a categorías fundamentalmente diferentes). La ontología social es una rama de la filosofía analítica que estudia la naturaleza y las propiedades de las entidades sociales. Ahora bien, la realidad social es generada por la acción intencional colectiva, continúa Bernal, y el derecho pertenece a la realidad social.

La herramienta teórica central del artículo de Bernal es la tesis de la práctica social; el derecho, entendido como un conjunto de normas válidas, está fundado en una práctica social. Una práctica social es concebida como un conjunto de acciones intencionales colectivas recurrentes. Una acción es tal, para Bernal, si se cumplen dos

3 Véase Shapiro, S., Legality, Cambridge, Harvard University Press, 2011, pág. 39 y ss. Cfr. también Weinberger, O., "Institutionalist Theories of Law", en Amselek, P. y MacCormick, N. (eds.), Controversies About Law's Ontology, Edinburgh, Edinburgh UP, 1991. 
condiciones: 1) que ella sea llevada a cabo por múltiples agentes individuales actuando conjuntamente como un grupo; 2) que los agentes individuales que actúan conjuntamente lo hagan de acuerdo con $-\mathrm{y}$ en razón de - ciertas intenciones comunes ['we-intentions'] $]^{4}$.

Austin, Hart y Shapiro proporcionan, de acuerdo con Bernal, tres teorías del derecho que ejemplifican la tesis general del derecho como entidad fundada en una práctica social así definida.

Algunos méritos y límites de la teoría de Austin se ponen eventualmente de manifiesto. Entre los límites, Bernal destaca el problema de la colectividad y el problema de la normatividad. Respecto del primero, el hábito general de obediencia que debe darse para definir al soberano de Austin no es necesariamente una acción colectiva. Puede hacerse de él tanto una lectura distributiva como una colectiva. Respecto del segundo problema, el hábito general de obediencia no logra capturar la normatividad del derecho, esto es, la capacidad de la autoridad del derecho de generar obligaciones jurídicas.

Luego Bernal se ocupa de la afirmación hartiana según la cual el derecho se funda en una práctica social normativa consistente en la aceptación de reglas sociales. Para Hart, la actitud de aceptar las reglas implica tres tipos de actividades sociales: el hábito de obediencia, el hábito de criticar las desviaciones y el hábito de tomar las críticas como legítimas. Pero una vez más encontramos el problema de la lectura distributiva de las actitudes relevantes, a saber: la aceptación de reglas de Hart, lo cual hace imposible explicar la dimensión social del derecho y la práctica jurídica. Por lo demás, para Bernal, en tanto que la teoría de Hart pone el foco de atención en los funcionarios jurídicos en particular (no es una condición necesaria para que haya un sistema jurídico que los ciudadanos también acepten las reglas), la caracterización del derecho como una amplia práctica oficial y no social torna a la teoría incapaz de explicar por qué tal práctica oficial da lugar a reglas que son jurídicamente vinculantes para toda la sociedad (el problema de la normatividad nuevamente).

4 Cf. en particular Searle, J. R., The Construction of Social Reality, New York, Free Press, 1995, págs. 23-26. 
Finalmente, Bernal hace observaciones sobre Shapiro y la teoría del derecho como plan. Los planes son normas y la actividad jurídica es una forma de planificación social centrada en el así llamado plan maestro. De acuerdo con Shapiro, la coerción no es una propiedad esencial del derecho; por otro lado, sostiene que el derecho tiene necesariamente un propósito moral porque el propósito fundamental de la actividad jurídica es solucionar las deficiencias morales de las circunstancias de legalidad. Para Bernal, Shapiro encara mejor que Austin y Hart los problemas mencionados, dado que la planificación social es necesariamente una actividad colectiva y dado que los requerimientos de la racionalidad instrumental junto con la tesis del propósito moral proporcionan una respuesta al problema de la normatividad. Como fuere, dice Bernal, dos preguntas críticas surgen sobre la teoría de Shapiro: 1) ¿requiere la práctica jurídica que cada una de las autoridades oficiales participe activamente mediante sus acciones individuales y sus intenciones cooperativas en las acciones del grupo relevantes para crear y ejecutar los planes? 2) ¿Es este concepto de plan apropiado frente a la preocupación por la normatividad del derecho? Con respecto a la primera cuestión, nos enfrentamos al problema de la alienación, esto es, la situación de participantes que acatan las reglas sin estar vinculados por ellas. Con respecto a la segunda cuestión, Bruno Celano ha argumentado recientemente ${ }^{5}$ que los planes (entendidos como Bratman lo hace, quien es la fuente inspiradora de Shapiro en esta materia) son producidos y aplicados por el agente mismo para su propia actuación y deliberación futuras (ellos son una herramienta de auto-administración), y por lo tanto no pueden ser usados para analizar el derecho y la normatividad jurídica, dado que la finalidad de la autoridad del derecho es establecer y aplicar planes para otros.

Bernal trata de rebatir estos ataques a la teoría y las afirmaciones de Shapiro, en particular, que la aceptación del plan maestro no es necesariamente explícita: ella puede ser tácita, y todavía puede

5 Véase Celano, B., "What can Plans do for Legal Theory?", en Canale, D. y Tuzet, G. (eds.), The Planning Theory of Law, London, Springer, 2013. 
fundarse en actividades colectivas (en parte intencionales y en parte no intencionales) ${ }^{6}$.

El artículo de Canale, "¿E1 Derecho está fundado en la Acción Colectiva?", pone de manifiesto algunas ventajas de la estrategia de Bernal en cuanto investigación acerca de la naturaleza del derecho, pero también algunos de sus inconvenientes. En particular, el hecho de que un cierto enfoque del derecho no encuadre con la tesis de la práctica social no prueba que tal enfoque sea inadecuado, ni que la tesis de la práctica social sea verdadera. Adicionalmente, de acuerdo con Canale, uno ha de notar que hay al menos dos modos de leer a los clásicos, como Austin y Hart: estratégicamente, por un lado, o de manera no comprometida, por otro, consistiendo lo primero en "renovar" el pensamiento de un autor en el marco de las cuestiones hodiernas, y consistiendo lo segundo en analizar un texto filosófico en sus propios términos y en su contexto. El enfoque de Bernal es estratégico, sostiene Canale, al tiempo que adoptando él mismo una actitud descomprometida afirma que una tal lectura de Austin, Hart y Shapiro pone en duda la tesis de la práctica social de Bernal, dado que sus teorías proveen algunos argumentos en favor de lo contrario, esto es, muestran que el derecho no posee necesariamente las características que Bernal considera que posee. De hecho, de acuerdo con Canale, el derecho posee algunos rasgos particulares que lo distinguen de otros fenómenos sociales (en particular, involucra especiales relaciones verticales y no constituye una actividad cooperativa, irenista, dado que abarca profundos desacuerdos y se vincula con el conflicto social).

Canale considera que el hábito de obediencia de Austin no puede ser traducido en la noción de acción intencional colectiva recurrente sin perder su significado y su significancia teórica, dado que su imagen del derecho es fundamentalmente no cooperativa y basada

${ }^{6}$ Esto me recuerda a la estrategia de Austin para defender la pretensión de que todo el derecho es mandato: el mortal contraejemplo del derecho consuetudinario es concebido como un mandato tácito del soberano. Véase Austin, J., The Province of Jurisprudence Determined, New York, The Nooday Press, 1954 [1832], págs. 30-32. Debo agregar que soy escéptico acerca de la fuerza argumentativa de tales estrategias re-definicionales. 
en relaciones verticales entre el soberano y los otros miembros de la sociedad política. En lo relativo al problema de la normatividad, Canale cree que el enfoque de Austin no es defectuoso, por cuanto proporciona una explicación psicológica y utilitaria de por qué las normas jurídicas guían la acción, y porque conecta esta explicación con el hábito general de obediencia.

Pasando a Hart, Canale subraya su explicación sociológica de la conformidad a las reglas y la afirmación; desde un punto de vista conceptual, hay una "relación interna" entre regla y práctica: en este sentido, de acuerdo con Hart, hablando estrictamente no es correcto decir que una práctica genera una regla, desde que participar en la práctica es seguir la regla pertinente o el conjunto de reglas, y viceversa. Esta suerte de argumento wittgensteiniano proporciona también una solución al problema de la normatividad (una solución deflacionaria, diría), dado que, para superarlo, es suficiente con especificar bajo qué condiciones la conducta humana puede ser apropiadamente descripta como gobernada por el derecho, haciendo abstracción de las efectivas intenciones de los participantes y de los destinatarios del derecho en particular. (Nótese que esta lectura de Hart es significativamente distinta de la lectura de Ekins, enfocada más bien en la actitud intencional de aceptación de reglas).

En cuanto a la visión de Shapiro y su apreciación por parte de Bernal, Canale sostiene que Bernal omite el carácter institucional del derecho reconocido por Shapiro como un rasgo distintivo. Este rasgo debilita una vez más la dimensión intencional del derecho, dado que Shapiro deja lugar para pensar acerca del derecho como una tecnología social impersonal que ayuda a resolver conflictos morales y sociales. Con excepción del plan maestro, en actividades masivas compartidas, las intenciones comunes pueden ser sustituidas por procedimientos comunes [we-procedures] expresados por normas tipo plan [plan-like norms]. En relación con el problema de la normatividad, Canale señala inter alia (utilizando un argumento formulado por Broome) que sobre la racionalidad instrumental solo puede darse una lectura de amplio alcance: la apreciación de la racionalidad no puede limitarse a medios, dado que incluye los fines. Por lo tanto, la adopción de 
un plan no es una condición suficiente para tener la obligación de llevarlo a cabo (de acuerdo al mencionado principio de la racionalidad instrumental): los planes no poseen la fuerza normativa del "deber", escribe Canale; más bien, ellos instancian requisitos de coherencia de medios-resultados los cuales son de ayuda en la evaluación de la acción humana. Por esta y otras razones puestas de manifiesto por Canale (incluida la objeción de Celano), Bernal no debería adoptar la teoría de los planes de Shapiro como una concepción adecuada de la práctica en la fundación del derecho.0

El artículo de Ekins, "Hechos, razones y acción colectiva: reflexiones en torno a la ontología social del derecho", valora positivamente la hipótesis de que el derecho es una especie de acción conjunta y una forma de realidad social, pero sostiene que no se trata solo de una realidad social: la realidad del derecho, de acuerdo con Ekins, ha de encontrarse en la razón y en los hechos, lo cual significa que la teoría del derecho debería esforzarse por explicar cómo es que hechos sociales pueden dar lugar a razones para la acción. Sin lugar a dudas, esto es lo que sucede cuando el derecho funciona correctamente o, en otras palabras, en el caso central de legalidad. Ekins piensa que Shapiro y Bernal no prestan suficiente atención a la agencia de grupo, dado que se centran, en cambio, en la acción conjunta simpliciter, sin atender a cómo un grupo se forma para actuar de un modo tal que evite la incoherencia o la inconsistencia, lo cual es el caso cuando el derecho funciona bien. Esta puede ser una falla en la mayor parte de la ontología social contemporánea, pero para Ekins no lo es en Tomás de Aquino ni en otros pensadores clásicos, como Hobbes, Locke y Rosseau, quienes muestran similar interés en la realidad social de la comunidad política, entendida como un grupo constituido por partes actuando conjuntamente.

Más en particular, más allá de su simpatía general por el enfoque de Bernal, Ekins tiene algunas objeciones para hacer a sus lecturas de Austin y Hart, y algunos desacuerdos - profundos, de hecho- con la teoría de Shapiro. Por ejemplo, Ekins afirma que no hay buenas razones para apoyar la lectura colectivista del hábito de obediencia de Austin. En Austin, dice Ekins, los sujetos del derecho no son un grupo 
que actúa, sino un grupo sobre el que se actúa (compárese en este punto la insistencia de Canale en las relaciones verticales de la práctica jurídica). Por el contrario, Ekins piensa que la lectura individualista de la aceptación no está abierta a discusión en el contexto hartiano, dado que la preocupación de Hart es acerca de las reglas sociales aceptadas por el grupo para el grupo.

Discutiendo luego a Shapiro, una preocupación mayor para Ekins tiene que ver con el problema de la alienación: la cuestión no es ni la posibilidad ni la existencia actual de participantes alienados, sino la importancia exagerada que Shapiro da a estas circunstancias. Ekins considera que el auto-entendimiento de los participantes comprometidos tiene prioridad explicativa en la reflexión acerca de la acción conjunta y la práctica jurídica. En pocas palabras, la actitud del participante alienado es explicativamente parasitaria respecto de la actitud del comprometido. Y ello involucra todavía una dimensión intencional, dado que la participación alienada en la acción conjunta involucra una intención de adoptar en parte el plan de acción conjunta dispuesto para todos los miembros. En sentido similar, el argumento de Bernal sobre la aceptación tácita subestima el caso central de aceptación intencional de reglas en un sistema jurídico bien formado y en correcto funcionamiento.

La réplica de Bernal, "Intencionalidad colectiva y estructura ontológica del derecho. Una respuesta a Richard Ekins y Damiano Canale", comienza por replantearse el propósito principal de su artículo previo: explorar en qué medida las teorías del derecho provistas por Austin, Hart y Shapiro establecen fundamentos apropiados para desarrollar la intuición de que el derecho posee la estructura ontológica (o la naturaleza) de una práctica social. Luego, Bernal apunta a la que considera la mayor objeción alzada por Canale y Ekins. En varios sitios dice haber sido mal interpretado por parte de sus dos comentaristas. Por ejemplo (contra Canale), niega haber tratado de evaluar a Austin y a Hart en base a los problemas teóricos contemporáneos. Y, aún más importante, a pesar de que pensaba -y piensa todavía - que la ontología social puede echar luz sobre nuestro entendimiento acerca de la naturaleza del derecho, no aprueba (contra 
Ekins) una reducción de la naturaleza del derecho a hechos sociales. Un punto interesante que surge de sus réplicas es la compatibilidad de la concepción de Bernal con una teoría no-positivista del derecho: él entiende que su enfoque no descarta la teoría del derecho natural ni prueba que el positivismo jurídico sea verdadero. Su "modesta" teoría es compatible con un enfoque (positivista) subjetivo de las intenciones de los participantes (ellas son lo que son, como hechos sociales) y también con un enfoque (no-positivista) objetivo (las intenciones deben ser apropiadas, desde un punto de vista normativo). En suma, si entiendo correctamente el punto de Bernal, el "proyecto de la intencionalidad jurídica" ["collective intentionality legal project'] posee un núcleo seminal que puede desarrollarse en diferentes direcciones.

Otro punto interesante es la discusión de la agencia de grupo y la acción representativa [proxy agency], donde la ontología de los grupos está en juego; Bernal sostiene (contra Ekins, tal como Bernal lo interpreta) que no hay necesidad de postular un agente por encima de los miembros de un grupo, dado que lo que el grupo hace es reducible a las intenciones y acciones de sus miembros individuales. También la lectura colectivista del hábito de obediencia de Austin y la lectura individualista de la actitud de aceptación de reglas de Hart pasan a primer plano, centrándose nuevamente en las intenciones individuales o comunes [I- or we-intentions] de los miembros del grupo en vistas a cimentar el "proyecto jurídico de la intención colectiva".

Dejo a los lectores la formación de sus propias opiniones acerca de quién está equivocado y quién tiene razón sobre estas cuestiones. Aquí solo deseo destacar un par de cosas buenas y una no tan buena, una preocupación.

En la totalidad de la discusión, desde mi punto de vista, hay al menos dos puntos generales que son muy positivos. Primero, es bueno perseguir los objetivos teóricos de la ontología social, en vistas a obtener una mejor comprensión de la naturaleza del derecho. Por el otro lado (como Canale resalta), es bueno también poner de manifiesto cuáles son los rasgos particulares del derecho, esto es, entender por qué el derecho no puede ser reducido a otras formas de realidad social. Segundo, es conceptualmente correcto sostener (tal como lo 
hace Bernal) que las reglas no son prácticas, y que no pueden ser reducidas a ellas: más bien, están fundadas en ellas. Confundirlas es incurrir en un error categorial. Esto es así dado que - a pesar del argumento de la "relación interna"- está claro que las propiedades de las reglas no son las propiedades de las prácticas, y viceversa.

Ahora la preocupación: una buena parte de las controversias hartianas (esto es, controversias acerca del modo apropiado de leer a Hart y, más que nada, acerca del modo de leer a los académicos que leen a Hart de uno u otro modo) corre el riesgo de resultar carente de interés. La pretensión de Hart de llevar a cabo una obra de "sociología descriptiva"7 es traicionada por aquellas hipersofisticadas disputas conceptuales que pierden las huellas de lo que sucede en el mundo real; los sistemas jurídicos están cambiando su forma dramáticamente si se los compara con el modelo del sistema jurídico nacional provisto de un bien definido catálogo jerarquizado de fuentes del derecho (regla de reconocimiento, en términos hartianos). Cambios dramáticos en el escenario internacional llaman a alguna revisión de nuestras categorías teóricas. Por lo tanto, la preocupación es que las categorías hartianas y las discusiones a este respecto se prueben no solo insuficientes, sino además irrelevantes y carentes de interés para lidiar con problemas contemporáneos vivos y pujantes en la práctica jurídica actual.

Como fuere, no debería leerse la presente discusión como un mero debate áulico sobre cuestiones académicas: lo más fecundo que puede hacerse es leerlo como un modo de aprehender mejor la naturaleza del derecho en el espíritu de la teoría general del derecho o Jurisprudence. De ser exitoso, esto inevitablemente echará luz sobre nuestra comprensión del fenómeno jurídico en el mundo real.

7 Hart, H. L. A., The Concept of Law, Oxford, Clarendon Press, 1994 [1961], pág. vi. 


\section{Bibliografía}

Austin, J., The Province of Jurisprudence Determined, New York, The Nooday Press, 1954 [1832].

Celano, B., "What can Plans do for Legal Theory?", en Canale, D. y Tuzet, G. (eds.), The Planning Theory of Law, London Springer, 2013.

Hart, H. L. A., The Concept of Law, Oxford, Clarendon Press, 1994 [1961].

Kelsen, H., "What is the Pure Theory of Law", Tulane Law Review, 34, 1960, págs. 269-276.

Searle, J. R., The Construction of Social Reality, New York, Free Press, 1995.

Shapiro, S. Legality. Cambridge, Harvard University Press, 2011.

Tuzet, G., "The Social Reality of Law", en Analisi e diritto, 2007, págs. 179-198.

Weinberger, O., "Institutionalist Theories of Law", en Amselek,

P. y MacCormick, N. (eds.), Controversies About Law's Ontology, Edinburgh, Edinburgh UP, 1991, págs. 43-53. 